SV

\title{
FORCED RESPONSE OF A SPINNING DISK UNDER SPACE-FIXED COUPLES
}

\author{
Jen-San Chen AND C.-M. Hsu \\ Department of Mechanical Engineering, National Taiwan University, Taipei, Taiwan 107, \\ Republic of China
}

(Received 22 January 1997, and in final form 30 April 1997)

\begin{abstract}
The forced response of a spinning disk under space-fixed couples is analyzed analytically using the eigenfunction expansion method. A general couple on the disk surface can be divided into two components, i.e., pitching (with moment axis in the radial direction) and rolling (with moment axis in the circumferential direction). Both the transient vibration and the steady state deflection of the spinning disk are presented, with emphasis on the effects of the rotation speed and external damping. Two types of external damping are considered; one is rotating with the disk and the other is space-fixed. It is found that in the sub-critical speed range, both the rotating and space-fixed dampings tend to suppress the transient response of a spinning disk under a space-fixed pitching couple. On the other hand, in the super critical speed range, the rotating damping tends to suppress the transient vibration while the spaced-fixed damping tends to destabilize the system.
\end{abstract}

(C) 1997 Academic Press Limited

\section{INTRODUCTION}

The spinning disk is at the heart of hard disks, floppy disks, and optical recording devices. Excessive disk vibrations degrade the signal from the read/write head and may destroy the disk by a "head crash". In the wood cutting industry, the undesirable vibration of the circular saw blade results in unacceptable waste of raw material. The proper design of reliable disk drives and efficient circular saws requires a thorough understanding of the mechanical characteristics of the spinning disk. The earliest investigation on the vibration of a spinning disk may be traced back to Lamb and Southwell [1], in which they derived the linearized equations of transverse deflection and examined the natural frequencies and mode shapes of the spinning disk. Prescott [2] gave a more cogent presentation of the results of Lamb and Southwell, and extended some of the analysis to disks with different clamping geometries and cross-sections.

Among many other research topics, the response of a spinning disk under point loads is particularly of interest because it is a decisive factor in the performance of high density disk drives. Mote [3] investigated the stability of a stationary annular plate subjected to circumferentially moving loads with harmonically varying amplitude. Weisensel and Schlack [4] extended Mote's work to include radially moving loads. It is noted that the problem of a stationary disk under a circumferentially moving load treated in [3] and [4] differs from the problem of a spinning disk under a space-fixed load by the centrifugal effect due to disk rotation. Although the differences between these two problems are not appreciable within certain ranges of rotation speed, it seems more straightforward to adopt 
the space-fixed co-ordinate system to describe the deflection of a spinning disk under space-fixed loads.

Benson and Bogy [5] investigated the steady state response of a spinning disk with extremely small bending rigidity under a space-fixed point force, and discussed the relations between the deflection pattern and the radial position of the loading point. Cole and Benson [6] later used an eigenfunction approach to extend the analysis in [5] to a spinning disk with mild bending rigidity. Ono and Maeno [7] calculated the response of a spinning floppy disk under space-fixed harmonic excitation. While these extensive studies on the steady state response and the response under harmonic excitation provide valuable physical insight into the behavior of disk deflection, very little information has been obtained on the vibrant transient response immediately following the excitation.

In a real-world floppy disk drive, the rotating disk is usually sandwiched by a pair of read/write heads. In the ideal case when these two heads line up perfectly, the resultant exerted by the heads onto the spinning disk is a single force. This is the situation investigated in the aforementioned work. On the other hand, when there exists slight misalignment between these two heads due to manufacturing or assembling errors, the resultant on the spinning disk will include a couple and a force. In the present paper we consider the response of a spinning disk under space-fixed couples. In the transient response analysis emphasis is given to the effects of the rotation speed on the deflection evolution immediately following the excitation of the couples. In view of the ever increasing operating speed in the current disk drive industry, this transient response may be crucial to the performance of the disk drives. Regarding the steady state response, we emphasize the different configurations when the disk is spinning below and beyond the critical speed. The effects of both the space-fixed as well as the rotating dampings on the amplitude of the response are also investigated.

\section{EQUATION OF MOTION}

Consider an elastic circular disk clamped on the inner radius $r=a$ and free on the outer radius $r=b$. The disk is spinning with constant rotation speed $\Omega$. The spinning disk is initially undeformed laterally until the application of a space-fixed concentrated couple at position $r=\xi, \theta=0$ at time $t=0$, as shown in Figure 1. The point couple can be divided into two components; the pitching couple $M_{r}$ with the axis in the radial direction and

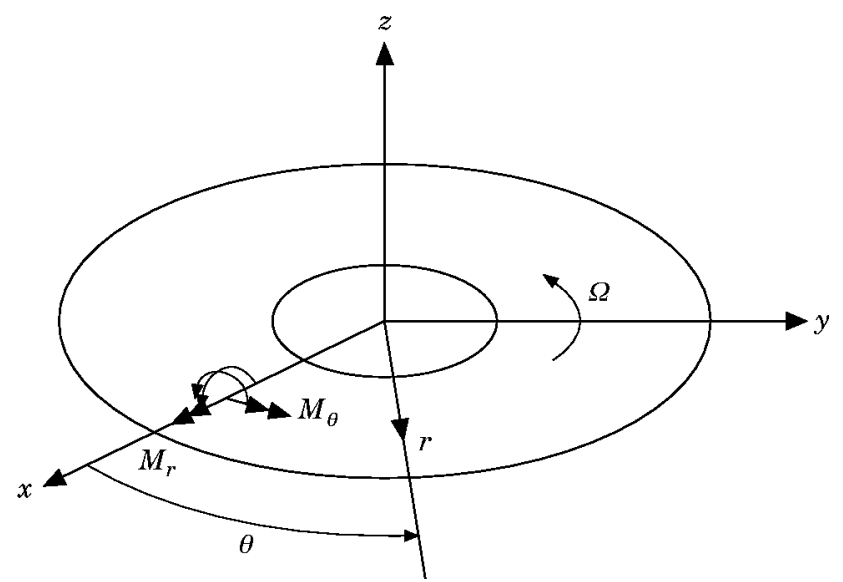

Figure 1. A spinning disk under space-fixed couples. 
rolling couple $M_{\theta}$ with the axis in the circumferential direction. The equation of motion of the spinning disk, in terms of transverse displacement $w$, and with respect to the stationary co-ordinate system $(r, \theta)$, can be written as

$$
\begin{gathered}
M w_{, t t}+(G+\hat{G}) w_{, t}+(K+\hat{K}) w= \\
{\left[\left(M_{\theta} / r\right) \delta^{\prime}(r-\xi) \delta(\theta)-\left(M_{r} / r^{2}\right) \delta(r-\xi) \delta^{\prime}(\theta)\right](f(t) / \rho h),}
\end{gathered}
$$

where operators $M, G, \hat{G}, K$, and $\hat{K}$ are defined as

$$
\begin{gathered}
M \equiv 1, \quad G \equiv 2 \Omega \partial / \partial \theta, \quad \hat{G} \equiv\left(c_{1}+c_{2}\right) / \rho h, \\
K \equiv \frac{D \nabla^{4}}{\rho h}+\Omega^{2} \frac{\partial^{2}}{\partial \theta^{2}}-\frac{1}{\rho r} \frac{\partial}{\partial r}\left(\sigma_{r} r \frac{\partial}{\partial r}\right)-\frac{\sigma_{\theta}}{\rho r^{2}} \frac{\partial^{2}}{\partial \theta^{2}}, \quad \hat{K} \equiv \frac{c_{1} \Omega}{\rho h} \frac{\partial}{\partial \theta} .
\end{gathered}
$$

The parameters $\rho, h$, and $D$ are the mass density, thickness, and flexural rigidity of the disk, respectively. Parameters $c_{1}$ and $c_{2}$ represent two types of homogeneous damping due to the surrounding air film; one of them, $c_{1}$, is spinning along with the disk, and the other, $c_{2}$, is space-fixed [8]. The axisymmetric in-plane stresses $\sigma_{r}$ and $\sigma_{\theta}$ in equation (5) are due to the centrifugal effect, and can be found in [8]. $\delta(:)$ is the Dirac delta function, and $\delta^{\prime}(:)$ is the derivative of $\delta(:)$ with respect to its parameter. $f(t)$ represents the loading history of the point couples. The initial conditions of the spinning disk are assumed to be $w(r, \theta)=0$ and $w_{, t}(r, \theta)=0$ at time $t=0^{-}$.

For a freely spinning disk (i.e., without point couples and external dampings) the equation of motion (1) can be reduced to

$$
M w_{, t}+G w_{, t}+K w=0 .
$$

The eigenvalues of the $\mathrm{e}^{\lambda t}$ time-reduced form of equation (7), together with the associated homogeneous boundary conditions, are purely imaginary and occur in complex conjugate pairs, i.e., $\lambda_{m n}= \pm \mathrm{i} \omega_{m n}$, where $\mathrm{i}=\sqrt{-1}$ and $\omega_{m n}$ is real. The eigenfunction corresponding to $\lambda_{m n}$ is in general complex and assumes the form

$$
w_{m n}=R_{m n}(r) \mathrm{e}^{\mathrm{i} n \theta} .
$$

The eigenfunction corresponding to $\bar{\lambda}_{m n}$ is $\bar{w}_{m n}$, where overbar means complex conjugate. It is noted that the eigenpairs $\left(\lambda_{m n}, w_{m n}\right)$ and $\left(\bar{\lambda}_{m n}, \bar{w}_{m n}\right)$ represent exactly the same physical mode. $w_{m n}$ in equation (8) with positive $n$ and positive $\omega_{m n}$ is a backward travelling wave with $n$ nodal diameters and $m$ nodal circles, which is also denoted by $(m, n)_{b}$. Similarly, $w_{m n}$ with negative $n$ and positive $\omega_{m n}$ is a forward travelling wave $(m,-n)_{f}$. The critical speed $\Omega_{c}$ for the mode $(m, n)$ is defined as the rotation speed at which $\omega_{m n}$ of the backward travelling wave $(m, n)_{b}$ becomes zero. For $\Omega$ greater than $\Omega_{c}$, the backward travelling mode appears to travel forward, and is called a "reflected wave", denoted by $(m, n)_{r}$. It is noted that for a reflected wave, the integer $n$ is considered as positive, while the natural frequency $\omega_{m n}$ is considered as negative. The radial function $R_{m n}(r)$ can be either solved in a power series form [9] or can be approximated by the nodal values obtained through a finite element procedure [8]. It is also noted that the eigenfunctions $w_{m n}$ are orthogonal with respect to the operators $M, G$, and $K$ [10],

$$
\begin{gathered}
\int_{0}^{2 \pi} \int_{a}^{b} \bar{w}_{m n} M w_{p q} r \mathrm{~d} r \mathrm{~d} \theta=\delta_{m p} \delta_{n q}, \quad \int_{0}^{2 \pi} \int_{a}^{b} \bar{w}_{m n} G w_{p q} r \mathrm{~d} r \mathrm{~d} \theta=\mathrm{i} 2 n \Omega \delta_{m p} \delta_{n q}, \\
\int_{0}^{2 \pi} \int_{a}^{b} \bar{w}_{m n} K w_{p q} r \mathrm{~d} r \mathrm{~d} \theta=\omega_{m n}\left(\omega_{m n}+2 n \Omega\right) \delta_{m p} \delta_{n q},
\end{gathered}
$$

where $\delta_{m p}$ is the Kronecker delta symbol. 


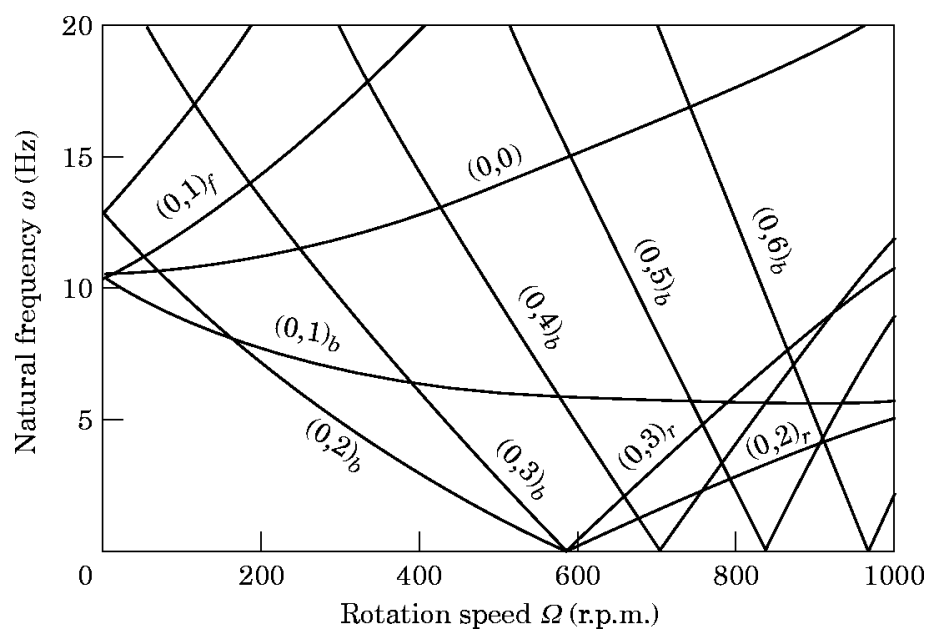

Figure 2. Natural frequencies of a flexible disk spinning from 0 to 1000 r.p.m.

Figure 2 shows the natural frequency loci of a spinning disk as the rotation speed varies [7]. The parameters used in the calculations are chosen to be representative of properties of a commercially available floppy disk; they are $\rho=1.3 \times 10^{3} \mathrm{~kg} / \mathrm{m}^{3}$, $D=2.1 \times 10^{-4} \mathrm{Nm}, h=0.078 \mathrm{~mm}, a=17.5 \mathrm{~mm}$ and $b=65 \mathrm{~mm}$. The critical speeds of the modes $(0,2)_{b},(0,3)_{b}$, and $(0,4)_{b}$ are 594, 589, and 708 r.p.m., respectively.

\section{EIGENFUNCTION EXPANSION METHOD}

The solution $w(r, \theta, t)$ of equation (1) can be expressed in terms of eigenfunctions $w_{m n}(r, \theta)$ as

$$
w(r, \theta, t)=\sum_{m=0}^{\infty} \sum_{n=-\infty}^{\infty}\left[c_{m n}(t) w_{m n}(r, \theta)\right]
$$

Both $c_{m n}(t)$ and $w_{m n}(r, \theta)$ in equation (12) are complex functions. However, it will be demonstrated later that after summation the displacement $w(r, \theta, t)$ in equation (12) is a real function. After substituting equation (12) into equation (1), multiplying both sides of equation (1) by $\bar{w}_{p q}$, integrating over the annular region and bearing in mind the orthogonal relations (9-11), one can discretize equation (1) into a system of decoupled equations for $c_{m n}(t)$

$$
\ddot{c}_{m n}+\left(2 \mathrm{i} n \Omega+\frac{c_{1}+c_{2}}{\rho h}\right) \dot{c}_{m n}+\left[\omega_{m n}\left(\omega_{m n}+2 n \Omega\right)+\frac{\mathrm{i} n c_{1} \Omega}{\rho h}\right] c_{m n}=F_{m n} f(t),
$$

where

$$
F_{m n}=(1 / \rho h)\left[-\mathrm{i} n M_{r}\left(R_{m n}(\xi)\right) / \xi+M_{\theta} R_{m n}^{\prime}(\xi)\right] .
$$

A superposed dot represents derivative with respect to time. The initial conditions for $c_{m n}$ are $c_{m n}(0)=0$ and $\dot{c}_{m n}(0)=0$. Recalling that at a certain rotation speed there are in general 
two modes with $m$ nodal circles and $n$ nodal diameters. In the sub-critical speed range, one is a forward mode and the other is a backward mode. In the super-critical speed range, one is a forward mode and the other is a reflected mode. In any case, if $\omega_{m n}$ is the natural frequency of one $(m, n)$ mode, $\left(\omega_{m n}+2 n \Omega\right)$ is the natural frequency of the other $(m, n)$ mode, also denoted by $\omega_{m \bar{n}}$. Consequently, equation (13) associated with these two modes can be rewritten as

$$
\ddot{c}_{m n}+\left(2 \mathrm{i} n \Omega+\left(c_{1}+c_{2}\right) / \rho h\right) \dot{c}_{m n}+\left[\omega_{m n} \omega_{m \bar{n}}+\mathrm{i} n c_{1} \Omega / \rho h\right] c_{m n}=F_{m n} f(t) .
$$

In other words, $c_{m n}(t)$ associated with these two $(m, n)$ modes are conjugate to each other. Since the mode shapes $w_{m n}$ of these two modes are also conjugate to each other, the contribution of these two modes in equation (12), will result in a real displacement $w(r, \theta, t)$.

One first considers the case when $f(t)=\delta(t)$. In this case the solution of equation (15) can be written as

$$
c_{m n}(t) / F_{m n}=\frac{\mathrm{H}(t) \mathrm{e}^{-\mathrm{i} \phi_{m n}}}{\sqrt{r_{m n}}}\left[\mathrm{e}^{\left(\alpha_{1 m n}+\mathrm{i} \beta_{1 m n}\right) t}-\mathrm{e}^{\left(\alpha_{2 m n}+\mathrm{i} \beta_{2 m n}\right)}\right],
$$

where $\mathrm{H}(t)$ represents the Heaviside step function, and

$$
\begin{gathered}
\phi_{m n}=\pi / 2+\frac{1}{2} \tan ^{-1}\left[4 n \Omega \rho h c_{2} /\left(-4 \rho^{2} h^{2} \hat{\omega}_{m n}^{2}+\left(c_{1}+c_{2}\right)^{2}\right)\right], \\
r_{m n}=\left\{\left[-4 \hat{\omega}_{m n}^{2}+\left(\left[c_{1}+c_{2}\right] / \rho h\right)^{2}\right]^{2}+\left(4 n \Omega c_{2} / \rho h\right)^{2}\right\}^{1 / 2} \\
\hat{\omega}_{m n}=\frac{1}{2}\left(\omega_{m n}+\omega_{m n}\right), \quad \alpha_{1 m n}=-\left(c_{1}+c_{2}\right) / 2 \rho h+\frac{1}{2} \sqrt{r_{m n}} \cos \phi_{m n}, \\
\alpha_{2 m n}=-\left(c_{1}+c_{2}\right) / 2 \rho h-\frac{1}{2} \sqrt{r_{m n}} \cos \phi_{m n}, \\
\beta_{1 m n}=-n \Omega+\frac{1}{2} \sqrt{r_{m n}} \sin \phi_{m n}, \quad \beta_{2 m n}=-n \Omega-\frac{1}{2} \sqrt{r_{m n}} \sin \phi_{m n} .
\end{gathered}
$$

It is noted that $\hat{\omega}_{m n}$ in equation (19) can be interpreted as the natural frequency of the $(m, n)$ mode as seen by an observer rotating with the disk.

One next considers the case when the spinning disk is subjected to a space-fixed step couple, $f(t)=\mathrm{H}(t)$. By using the solution for the impulsive excitation, equation (16), and the convolution integral, the solution $c_{m n}(t)$ of equation (15) under a step couple with dampings included can be written as

$$
\frac{c_{m n}(t)}{F_{m n}}=\frac{\mathrm{e}^{-\mathrm{i} \phi_{m n}}}{\sqrt{r_{m n}}}\left[\frac{\mathrm{e}^{\left(\alpha_{1 m n}+\mathrm{i} \beta_{1 m n}\right) t}-1}{\alpha_{1 m n}+\mathrm{i} \beta_{1 m n}}-\frac{\mathrm{e}^{\left(\alpha_{2 m n}+\mathrm{i} \beta_{2 m n}\right) t}-1}{\alpha_{2 m n}+\mathrm{i} \beta_{2 m n}}\right] .
$$

Substituting equation (24) into equation (12) and rearranging the series, one obtains the expression for the displacement of the spinning disk under space-fixed pitching couple $M_{r} \mathrm{H}(t)$ as

$$
\begin{aligned}
w(r, \theta, t)= & \sum_{m=0}^{\infty} \sum_{n=1}^{\infty} \frac{2 n M_{r} R_{m n}(\xi) R_{m n}(r)}{\rho h \xi \sqrt{r_{m n}}}\left[1 /\left(\alpha_{1 m n}^{2}+\beta_{1 m n}^{2}\right)\left(\alpha_{1 m n} \mathrm{e}^{\alpha_{1 m n} t} \sin \left(\beta_{1 m n} t-\hat{\phi}_{m n}\right)\right.\right. \\
& \left.+\alpha_{1 m n} \sin \hat{\phi}_{m n}-\beta_{1 m n} \mathrm{e}^{\alpha_{1 m n} t} \cos \left(\beta_{1 m n} t-\hat{\phi}_{m n}\right)+\beta_{1 m n} \cos \hat{\phi}_{m n}\right) \\
& +1 /\left(\alpha_{2 m n}^{2}+\beta_{2 m n}^{2}\right)\left(-\alpha_{2 m n} \mathrm{e}^{\alpha_{2 m n} t} \sin \left(\beta_{2 m n} t-\hat{\phi}_{m n}\right)-\alpha_{2 m n} \sin \hat{\phi}_{m n}\right. \\
& \left.\left.+\beta_{2 m n} \mathrm{e}^{\alpha_{2 m n} t} \cos \left(\beta_{2 m n} t-\hat{\phi}_{m n}\right)-\beta_{2 m n} \cos \hat{\phi}_{m n}\right)\right]
\end{aligned}
$$


where

$$
\hat{\phi}_{m n}=\phi_{m n}-n \theta .
$$

In the special case when damping does not exist, equation (25) can be reduced to

$$
\begin{aligned}
w(r, \theta, t) & =\sum_{m=0}^{\infty} \sum_{n=1}^{\infty} \frac{2 n M_{r} R_{m n}(\xi) R_{m n}(r)}{\xi \rho h} \\
& \times\left\{\frac{-\sin n \theta}{\omega_{m n} \omega_{m \bar{n}}}+\frac{1}{2 \hat{\omega}_{m n}}\left[\frac{\sin \left(n \theta+\omega_{m n} t\right)}{\omega_{m n}}+\frac{\sin \left(n \theta-\omega_{m \bar{n}} t\right)}{\omega_{m \bar{n}}}\right]\right\} .
\end{aligned}
$$

Since $n$ in equations (25) and (27) is restricted to being positive, $\omega_{m \bar{n}}$ always represents the natural frequency of the forward travelling mode. From equation (27) one can see that the response under a space-fixed step couple includes the contributions from all travelling modes with respective natural frequencies, together with a term independent of time. More importantly, the sign of this time-independent term depends on the product $\omega_{m n} \omega_{m \bar{n}}$, which is positive in the subcritical speed range, and is negative in the supercritical speed range. This term also corresponds to the steady state deformation when appropriate damping mechanism is introduced.

For the case when the spinning disk is under a space-fixed rolling couple $M_{\theta} \mathrm{H}(t)$, the displacement field can be derived as

$$
\begin{aligned}
w(r, \theta, t)= & \sum_{m=0}^{\infty} \sum_{n=0}^{\infty} \frac{\gamma_{n} M_{\theta} R_{m n}^{\prime}(\xi) R_{m n}(r)}{\rho h \sqrt{r_{m n}}} \\
& \times\left[\frac{1}{\alpha_{1 m n}^{2}+\beta_{1 m n}^{2}} \times\left(-\alpha_{1 m n} \mathrm{e}^{\alpha_{1 m n} t} \cos \left(\beta_{1 m n} t-\hat{\phi}_{m n}\right)\right.\right. \\
& \left.+\alpha_{1 m n} \cos \hat{\phi}_{m n}-\beta_{1 m n} \mathrm{e}^{\alpha_{1 m n} t} \sin \left(\beta_{1 m n} t-\hat{\phi}_{m n}\right)-\beta_{1 m n} \sin \hat{\phi}_{m n}\right) \\
& +\frac{1}{\alpha_{2 m n}^{2}+\beta_{2 m n}^{2}}\left(\alpha_{2 m n} \mathrm{e}^{\alpha_{2 m n} t} \cos \left(\beta_{2 m n} t-\hat{\phi}_{m n}\right)-\alpha_{2 m n} \cos \hat{\phi}_{m n}\right. \\
& \left.\left.+\beta_{2 m n} \mathrm{e}^{\alpha_{2 m n} t} \sin \left(\beta_{2 m n} t-\hat{\phi}_{m n}\right)+\beta_{2 m n} \sin \hat{\phi}_{m n}\right)\right]
\end{aligned}
$$

where $\gamma_{0}=1$, and $\gamma_{n}=2$ when $n \neq 0$.

It is well known that the classical plate theory is somewhat anomalous in transient response analysis since it does not yield the expected wave front separating disturbed and undisturbed regions. This phenomenon can also be observed in equations (25) and (28), which show that the whole disk "feels" the excitation instantly after the excitation. This is related to the fact that classical plate theory asserts that the wave speed of a disturbance approaches infinity as the associated wave length approaches zero. It is noted that, however, as time increases the displacement at any point with distance $d$ away from the origin of the point disturbance increases in spatial wave length. By assuming that classical plate theory may become reliable when the wave length becomes 16 times longer than the plate thickness, Medick [11] estimated a critical time interval in the order of $2 d \sqrt{E / \rho}$, which is $0.06 \mathrm{~ms}$ in the present case. In other words, the transient analysis based on classical plate theory should be reliable after this critical time interval. For more accurate 
response immediately after the excitation, higher order plate theory considering the effect of rotary inertia and shear deformation should be employed.

\section{TRANSIENT RESPONSE WITHOUT DAMPING}

In this section the transient response of the spinning disk under space-fixed couples without damping effect is considered. Figure 3 shows the slope-couple ratio history at the load point as the flexible disk is under step pitching couple $M_{r}$. The number of modes used in expansion (27) is restricted to $m<5$ and $n<30$. The loading position $\xi / b$ is chosen to be 0.54 . $\Omega=360$ r.p.m. is the operating speed of most floppy disk drives. It is observed that the mean slope and the amplitude of the slope variation increase as the rotation speed increases from 360 to 580 r.p.m. In particular, the response becomes unbounded as the rotation speed approaches the first critical speed. It is also noted that the slope $(1 / r)(\partial w / \partial r)$ at the load point is always in the same sense as that of the point couple when the rotation speed is in the subcritical speed range. As the rotation speed continues to increase beyond the critical speeds, the influences of the reflected waves $(0,2)_{r}$ and $(0,3)_{r}$ dominate the transient response, and the slope at 610 r.p.m. is primarily opposite to the sense of the point couple. As the rotation speed continues to increase the influence of another backward wave $(0,4)_{b}$ kicks in while the influences of the reflected waves diminish. Consequently, the mean slope changes from negative to positive value as the rotation speed increases from the critical speeds of modes $(0,2)$ and $(0,3)$ toward the critical speed of mode $(0,4)$, as shown by the slope curve corresponding to 700 r.p.m. The dominant periods of these transient responses are correspondent to the lowest natural frequencies of the disk spinning at respective rotation speeds. For instance, the dominant periods for $\Omega=360$ and 550 r.p.m. are 0.27 and $1.69 \mathrm{~s}$, respectively.

The irregular zigzags in the load-point slope curves of Figure 3 may be considered as being due to the superposition of many modes with different natural frequencies. It may also be considered as the result of complex continuous superposition of many waves that reflect from the free outer rim and the clamped inner rim, and the waves travelling in both directions circumferentially. These two views are actually equivalent, with one from the

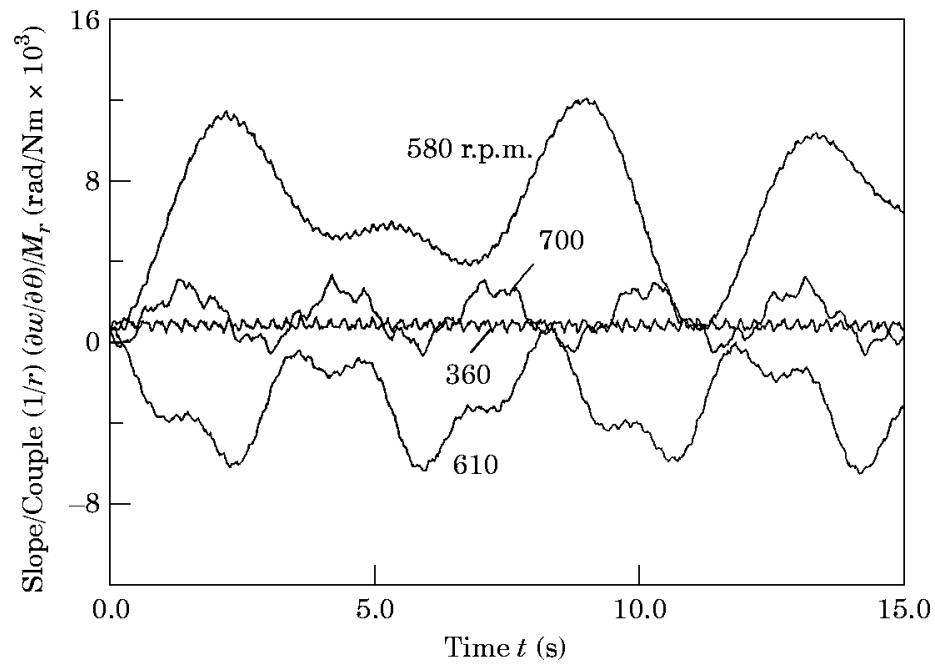

Figure 3. Slope-couple ratio history at the load point when the spinning disk is under a step pitching couple, $c_{1}=c_{2}=0 \cdot 0 \mathrm{Ns} / \mathrm{m}^{3}$. 

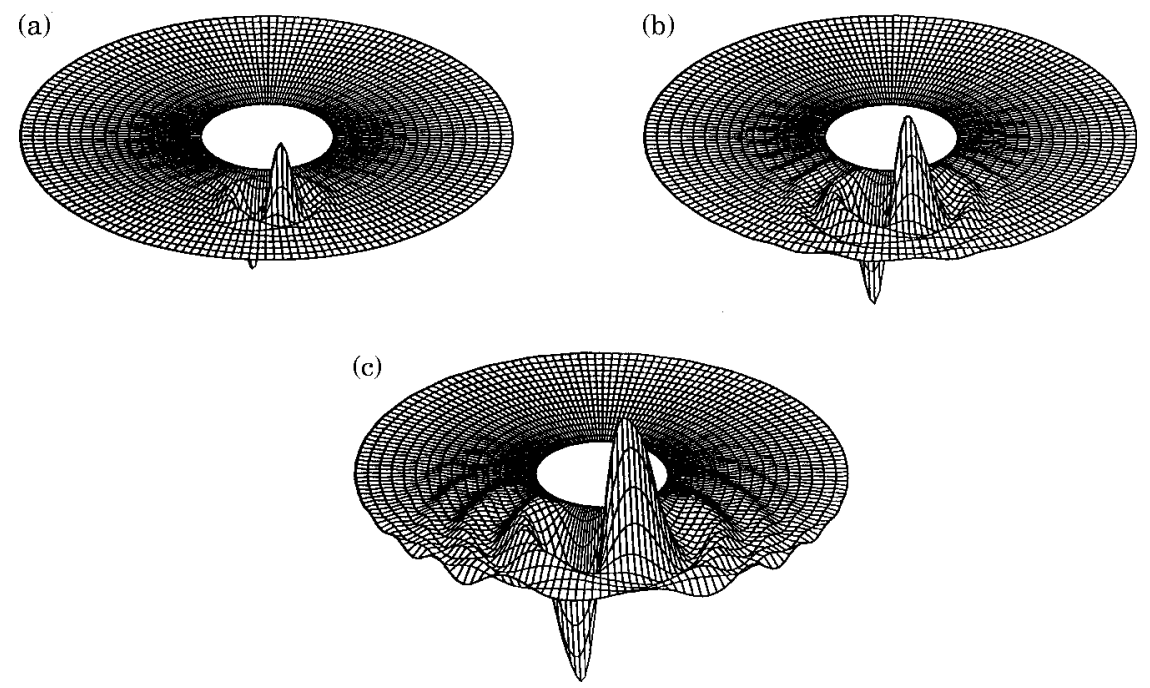

Figure 4. Deformation evolution of a disk under step pitching couple and spinning at 360 r.p.m., scale $=0 \cdot 02$. Times (ms): (a) $0 \cdot 2$; (b) $0 \cdot 4$; (c) $0 \cdot 8$.

frequency domain viewpoint and the other from the time domain viewpoint. It is noteworthy that a similar phenomenon can also be observed in the transient response of a transporting tape under a space-fixed point load [12].

Figure 4 shows the deformed shape at various time steps before $0.8 \mathrm{~ms}$ with a scale factor 0.02 for a disk spinning at 360 r.p.m. A black dot denotes the position of the space-fixed couple. From Figure 4 one can observe the wave propagation phenomenon in detail. It is noted that the deformation pattern is almost anti-symmetric with respect to the loading point while the disk is rotating in the counterclockwise sense. Calculations at 0 and 610 r.p.m. also show similar anti-symmetric deformation pattern with different amplitudes at the early state of the loading. In other words, rotation does not have significant effect in the deformation pattern at the very early stage of the excitation as the rotation speed is up to the range of the first critical speed.

It is expected that the anti-symmetric pattern of the deformation would be destroyed when the convective velocity of the rotating media is significant compared to the phase speed of the propagating wave. The authors estimate roughly the rotation speed at which the convective velocity $b \Omega$ of the disk is comparable to the phase velocity of a wave with wave length equal to $1 / 15$ of the outer circumference of the disk as

$$
\Omega=\left(15 / b^{2}\right) \sqrt{D / \rho h}
$$

which is about 9950 r.p.m. in the current case. Therefore, the convective velocity of the media for the disk rotating at 360 r.p.m. is about $\frac{1}{36}$ of the phase velocity of the plate. In Figure 5 the rotation speed is raised up 5100 r.p.m. and the deformation evolution before $0.8 \mathrm{~ms}$ is recorded. Obvious asymmetry can be observed in the deformation pattern. In particular, waves tend to accumulate in the upstream region.

Benson and Bogy [5] defined a dimensionless parameter $\alpha=8 D /\left[\rho \Omega^{2} b^{4}(3+v)\right]$ to characterize the bending stiffness of the spinning disk. A small $\alpha$ means that the effect of the fourth order bending operator in equation (5) is relatively small compared to the contribution from the membrane effect due to the centrifugal stress. For the disk rotating at 360 and 5100 r.p.m., the parameter $\alpha$ is 0.2 and 0.001 , respectively. Therefore, the 

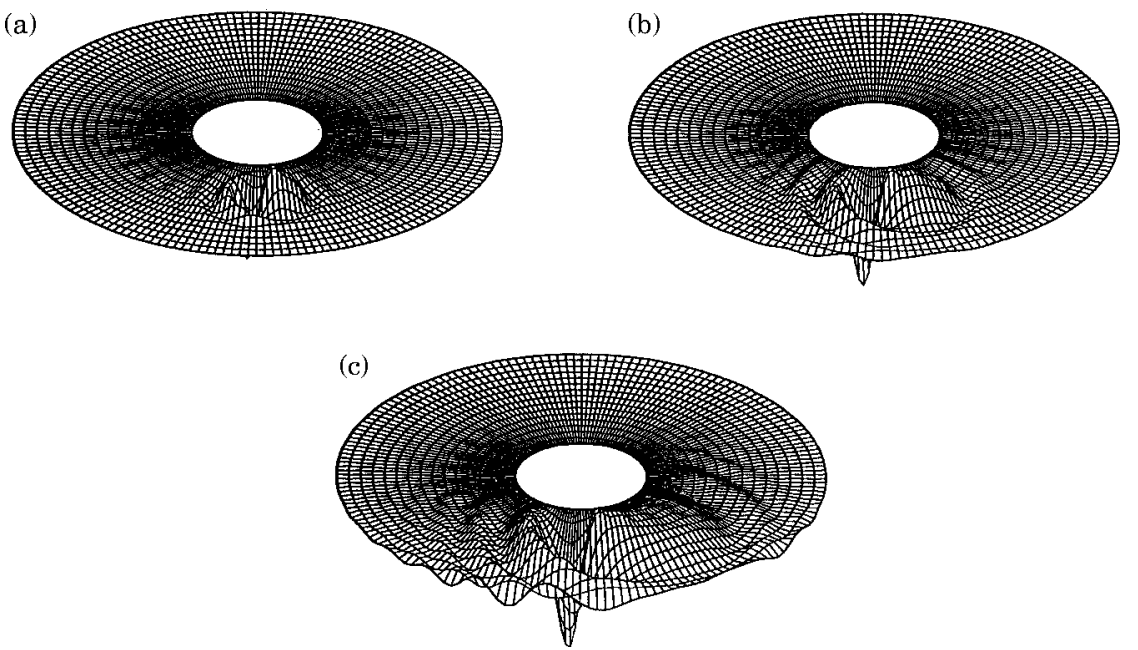

Figure 5. Deformation evolution of a disk under step pitching couple and spinning at 5100 r.p.m., scale $=0 \cdot 02$. Times (ms): (a) $0 \cdot 2$; (b) $0 \cdot 4$; (c) $0 \cdot 8$.

deformation pattern in Figure 5 is characteristic of a "membrane-like" disk in the sense that the membrane effect becomes dominant compared to the bending effect of the disk.

Figures 6 and 7 show the deformation evolution before $0.8 \mathrm{~ms}$ when the disk is subjected to the step rolling couple $M_{\theta} \mathrm{H}(t)$, and when it rotates at 360 and 5100 r.p.m., respectively.

\section{DAMPING EFFECTS AND STEADY STATES}

It is in general difficult to estimate the damping values of the surrounding air on the spinning disk. In the following calculations one assumes that the values of rotating damping $c_{1}$ and space-fixed damping $c_{2}$ are 0.1 and $0.5 \mathrm{Ns} / \mathrm{m}^{3}$, respectively [8]. Figure 8 shows the slope history at the loading point when the disk is subjected to pitching couple
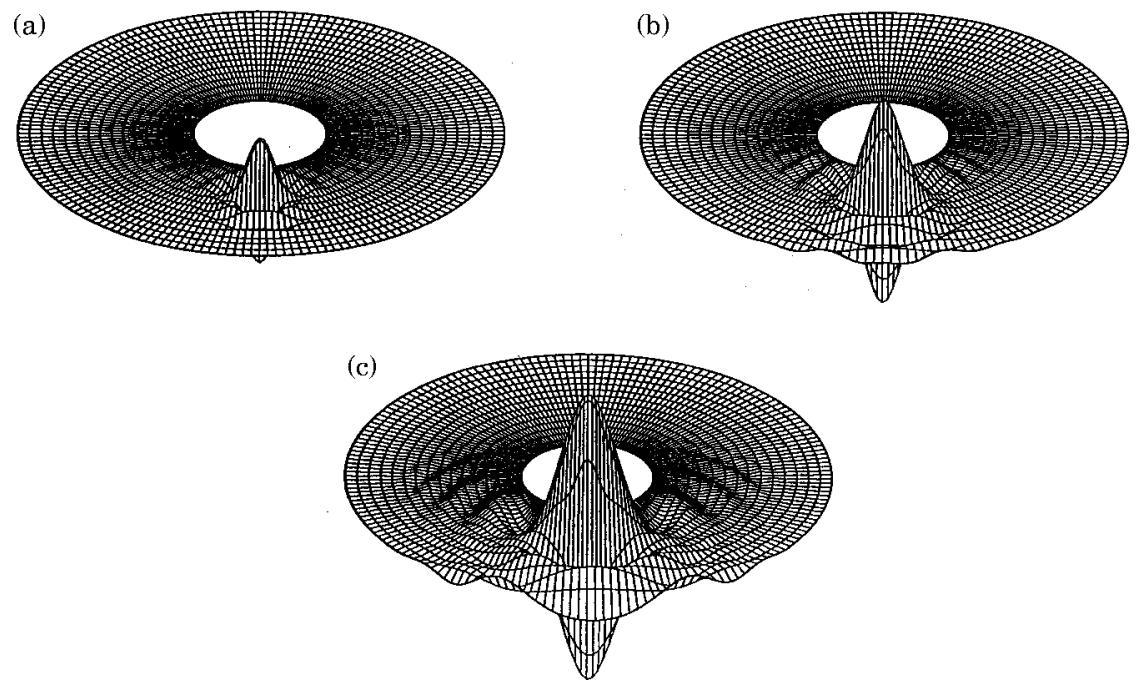

Figure 6. Deformation evolution of a disk under step rolling couple and spinning at 360 r.p.m., scale $=0 \cdot 02$. Times (ms): (a) $0 \cdot 2$; (b) $0 \cdot 4$; (c) $0 \cdot 8$. 

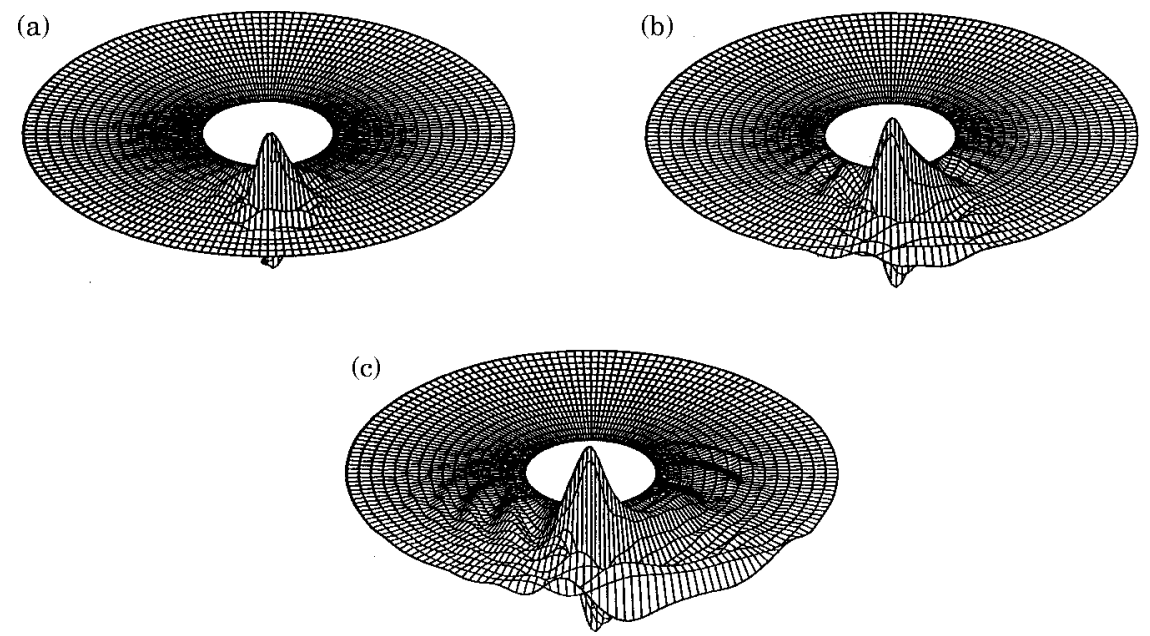

Figure 7. Deformation evolution of a disk under step rolling couple and spinning at 5100 r.p.m., scale $=0 \cdot 03$. Times (ms): (a) $0 \cdot 2$; (b) $0 \cdot 4$; (c) $0 \cdot 8$.

and only $c_{1}$ is included in the calculation. It is observed that the tiny zigzags in the slope curves are smoothed out, and the maximum overshoot decreases significantly compared to the undamped case in Figure 3. The deformations appear to reach their steady states within $8 \mathrm{~s}$ in both the sub- and supercritical speed range. The steady state deformation patterns at speeds 580 and 610 r.p.m., one below and the other above the critical speed, are shown in Figure 9 with the scale factor being 0.0001. The dominant components in these two steady state deformations are both the $(0,2)$ mode.

Figure 10 shows the slope history at the load point when the disk is under pitching couple and only $c_{2}$ is included. For the sub-critical speeds, 360 and 580 r.p.m., the amplitudes of the slope curves tend to settle to the steady state values as time goes by. For the supercritical speeds, 610 and 700 r.p.m., however, the amplitudes tend to grow unboundedly. It is particularly obvious for the slope curve corresponding to 700 r.p.m. These calculations suggest that in the subcritical speed range, both the rotating and space-fixed damping tend to suppress the transient response of a spinning disk under a space-fixed pitching couple. On the other hand, in the supercritical speed range, the

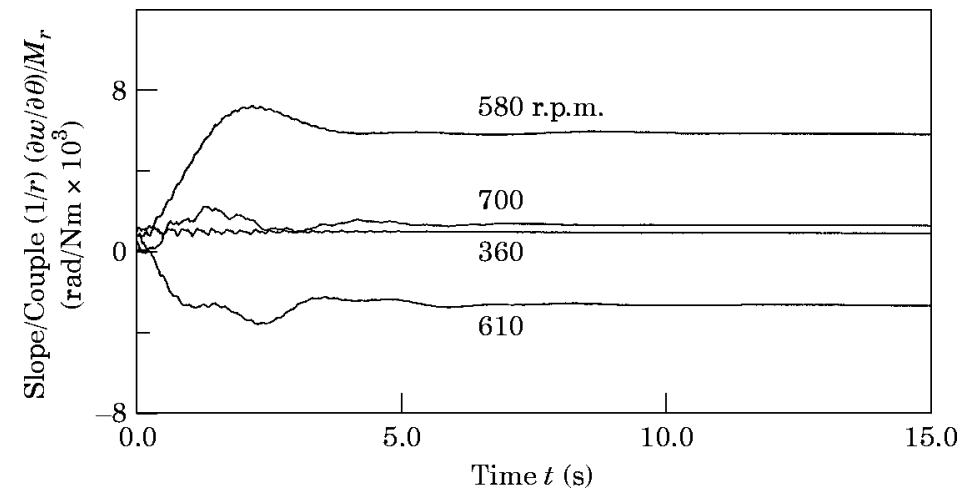

Figure 8 . Slope-couple ratio history at the load point when the disk is under pitching couple and rotating damping $c_{1}=0 \cdot 1 \mathrm{Ns} / \mathrm{m}^{3}$ is included. $c_{2}=0 \cdot 0 \mathrm{Ns} / \mathrm{m}^{3}$. 

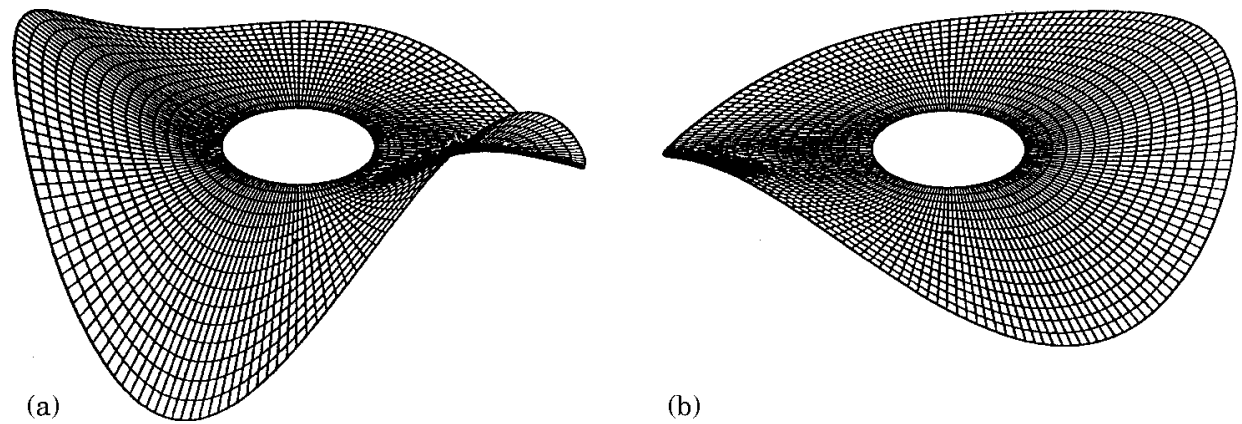

(b)

Figure 9. Steady state of the spinning disk at scale $=0.0001$ under pitching couple and spinning at (a) 580 and (b) 610 r.p.m.

rotating damping tends to suppress the transient vibration while the space-fixed damping tends to destabilize the system.

In order for the displacement to settle to a steady state value, it is necessary for the exponents in equations (25) and (28) to be negative, i.e., $\alpha_{1 m n}<0$ and $\alpha_{2 m n}<0$. These two conditions can lead to the relation.

$$
\sqrt{r_{m n}}\left|\cos \phi_{m n}\right|<\left(c_{1}+c_{2}\right) / \rho h
$$

Further simplification reduces equation (30) to

$$
\left(n \Omega c_{2} /\left[c_{1}+c_{2}\right]\right)^{2}<\hat{\omega}_{m n}^{2}
$$

In the case when $c_{1}>0$ and $c_{2}=0$, equation (31) reduces to

$$
0<\hat{\omega}_{m n}^{2}
$$

Therefore, transient vibration is always suppressed by the rotating damping in both the sub- and supercritical speed ranges. In the other case when $c_{1}=0$ and $c_{2}>0$, equation (31) reduces to

$$
0<\omega_{m n} \omega_{m \bar{n}}
$$

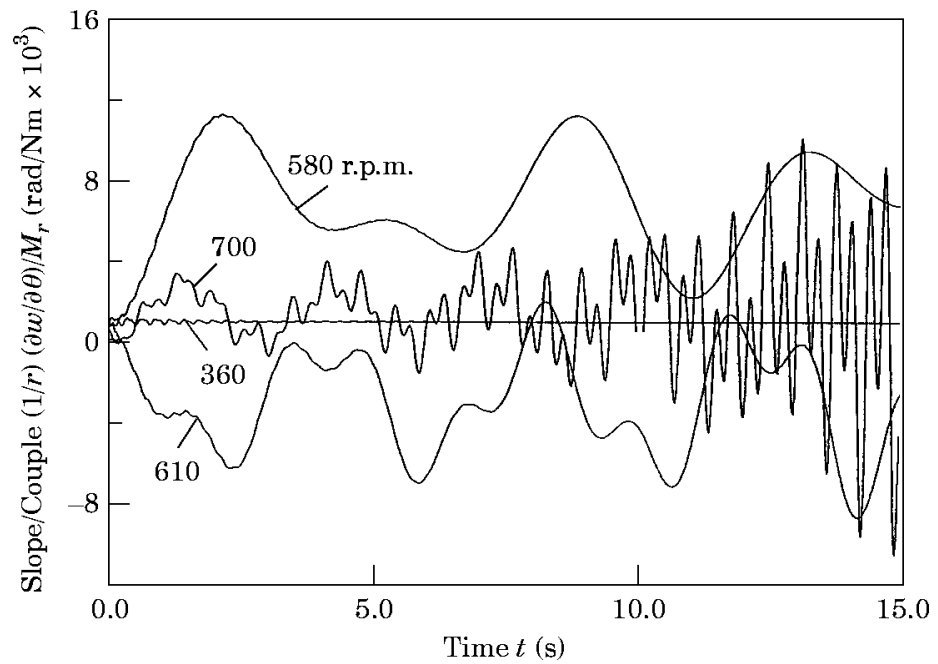

Figure 10. Slope-couple ratio history at the load point when the disk is under pitching couple and space-fixed damping $c_{2}=0.5 \mathrm{Ns} / \mathrm{m}^{3}$ is included. $c_{1}=0.0 \mathrm{Ns} / \mathrm{m}^{3}$. 
In the sub-critical speed range, the product $\omega_{m n} \omega_{m \bar{n}}$ is positive and the deflection of the spinning disk will eventually settle to a steady state. On the other hand, this product is negative in the supercritical speed range. The exponents in equations (25) and (28) are consequently positive and the deflection of the spinning disk tends to grow unboundedly.

\section{CONCLUSIONS}

In this paper the closed form solution for the forced response of a spinning disk under space-fixed couples has been derived. Both pitching and rolling couples are considered. In the case when the convective speed of the rotating media is negligible compared to the

phase speed of the propagating wave, the deformation pattern in the early stage of excitation under pitching couples appears anti-symmetric with respect to the radius passing through the load point. When the disk rotates at high speed such that the convective velocity is comparable to the wave speed, this anti-symmetric pattern is destroyed.

The effects of external damping are also investigated. It is found that the rotating damping always suppresses the transient response of the spinning disk. On the other hand, the space-fixed damping stabilizes the transient response only when the disk rotates in the subcritical speed range. When the disk rotates in the supercritical speed range, the space-fixed damping always destabilizes the system.

\section{ACKNOWLEDGMENT}

The results presented here were obtained in the course of research supported by a grant from the National Science Council of the Republic of China.

\section{REFERENCES}

1. H. LAmb and R. V. Southwell 1921 Proceedings of the Royal Society 99, 272-280. The vibration of a spinning disk.

2. J. Prescott 1961 Applied Elasticity. New York: Dover Publications; Chapter 18.

3. C. D. Mote 1970 Journal of the Franklin Institute 290, 329-344. Stability of circular plates subjected to moving loads.

4. G. N. WeisEnSEL and A. L. SCHLACK 1993 American Society of Mechanical Engineers Journal of Applied Mechanics 60,649-661. Response of annular plates to circumferentially and radially moving loads.

5. R. C. Benson and D. B. Bogy 1978 American Society of Mechanical Engineers Journal of Applied Mechanics 45, 636-642. Deflection of a very flexible spinning disk due to a stationary transverse load.

6. K. A. Cole and R. C. Benson 1988 American Society of Mechanical Engineers Journal of Applied Mechanics 55, 453-457. A fast eigenfunction approach for computing spinning disk deflections.

7. K. Ono and T. Maeno 1987 Tribology and Mechanics of Magnetic Storage Systems 3, SP.21, (STLE), 144-151. Theoretical and experimental investigation on dynamic characteristics of a 3.5-inch flexible disk due to a point contact head.

8. K. Ono, J.-S. Chen and D. B. Bogy 1991 American Society of Mechanical Engineers Journal of Applied Mechanics 58, 1005-1014. Stability analysis for the head-disk interface in a flexible disk drive.

9. G. Adams 1987 International Journal of Mechancial Sciences 29, 525-531. Critical speeds for a flexible spinning disk.

10. J.-S. Chen and C.-C. Wong 1996 Journal of the Chinese Society of Mechanical Engineers 17, 251-259. Modal interactions in a spinning disk on a floating central collar and restrained by multiple springs.

11. M. A. Medick 1961 American Society of Mechanical Engineers Journal of Applied Mechanics 28, 223-228. On classical plate theory and wave propagation. 
12. S. MüFTü and R. C. BENSON 1994 American Society of Mechanical Engineers Journal of Vibration and Acoustics 116, 567-572. A numerical solution for the transient displacement of a circumferentially moving cylindrical shell. 